\title{
3D perception of numerical hologram reconstructions enhanced by motion and stereo
}

\author{
Risto Näsänen \\ RFMedia Laboratory \\ University of Oulu \\ Ylivieska, Finland \\ risto.nasanen@oulu.fi, risto.nasanen@iki.fi \\ Thomas J. Naughton \\ Department of Computer Science \\ National University of Ireland \\ Maynooth, Ireland and \\ RFMedia Laboratory \\ University of Oulu \\ Ylivieska, Finland
}

\author{
Tristan Colomb and Yves Emery \\ Lyncee Tec Inc. \\ Lausanne, Switzerland
}

\begin{abstract}
We investigated the question of how the perception of 3D information of digital holograms reconstructed numerically and presented on conventional displays depends on motion and stereoscopic presentation. Perceived depth in an adjustable random pattern stereogram was matched to the depth in holographic objects. The objects in holograms were a microscopic biological cell and a macroscopic coil. Stereoscopic presentation increased perceived depth substantially in comparison to non-stereoscopic presentation. When stereoscopic cues were weak or absent e.g. because of blur, motion increased perceived depth considerably. However, when stereoscopic cues were strong, the effect of motion was small. In conclusion, for the maximisation of perceived 3D information of holograms on conventional displays, it seems highly beneficial to use the combination of motion and stereoscopic presentation.
\end{abstract}

\section{INTRODUCTION}

Digitally recorded holograms can be reconstructed numerically or alternatively optoelectronically. In principle, optoelectronic reconstruction allows one to view the depicted object or scene from different angles and accommodate (focus) to different depths in the reconstruction. Similarly, by using numerical reconstruction it is possible to compute different angular views of the hologram to different depths. While writing this paper, the development of the optoelectronic holographic display technology is still in its early stages. Therefore, before this technology becomes more matured, displaying numerical reconstructions on conventional stereo or non-stereo displays seems to be a useful alternative.

Any single numerical hologram reconstructions can only contain monocular depth information, such as texture gradients and linear perspective, and shading. The use of multiple numerical reconstructions of different perspectives and reconstruction depths allows one to extract much more of the
3D information of holograms, however. Since conventional stereo displays are far from being truly three-dimensional it is of interest to investigate the general question of how the information of multiple reconstructions should be presented on these displays to optimise 3D perception. For example, one way of combining hologram reconstructions, suggested by Lehtimäki and Naughton [1], is to generate two reconstructions that form a stereo pair so that one of them is near-focused and one far-focused. Because of binocular fusion this approach gives a single perception of increased depth of focus combined with stereoscopic depth. In general, the knowledge of the relationship between information presentation and perception will allow the design of better ways of mediating the depth information of hologram reconstructions on conventional displays.

The specific purpose of this study was to investigate the question of how the perception of depth in hologram reconstructions depends on the availability of stereoscopic and motion depth cues. We used non-stereoscopic and stereoscopic images with and without motion. The paper shows that by using multiple numerical reconstructions presented as combinations of motion and stereo pairs indeed can significantly enhance the mediation of holographic depth information to the viewer.

\section{METHODS}

\section{A. Stereoscopic depth matching tool}

A random pattern stereoscopic software tool was developed for the estimation of perceived depth in objects and images. The tool consisted of a random pattern noise field. The random field had two rectangular areas. The stereoscopic depth of the rectangular areas could be adjusted by using graphical sliders. The depth was produced so that the position of the right eye image was changed either to the right or left. 
A rightward change (positive disparity) produced depth behind the display surface and a leftward change (negative disparity) produced depth in front of the display surface. By means of two graphical sliders the observer adjusted the stereoscopic depth of the upper and lower rectangles so that they corresponded to the perceived depth of certain points (typically the near edge and far edge) in the images displayed beside the tool.

\subsection{Hologram sequences}

One sequence of holograms was macroscopic and one microscopic. In the macroscopic hologram sequence, the object was a small electronic coil. The coil object was captured at different angles by rotating the object in depth about the vertical axis from left to right in steps of $3 \mathrm{deg}$. The video was trimmed to 23 frames and shown at a rate of 15 frames per second and looped back and forth continuously during the perceptual task. The amplitude of the rotational movement was $73 \mathrm{deg}$. The size of the reconstruction was 512 x 512 pixels, which corresponds to $13.8 \times 13.8 \mathrm{~cm}^{2}$ and $8.8 \mathrm{x}$ $8.8 \mathrm{deg}^{2}$ of visual angle at a viewing distance of $90 \mathrm{~cm}$.

The microscopic object was a K562 leukaemia cell captured by a transmission digital holographic microscope (DHM T1000 ${ }^{\circledR}$ from Lyncée Tec). The cell, surrounded by physiological medium, was maintained in a dielectric field cage and was rotated using dielectrophoretic motion [2]. The recorded hologram, corresponding to the interference between a reference wave and the optical wave passing through the cell, is processed to reconstruct the cell depth map for each orientation $[3,4]$. The video was trimmed to 8 frames and shown at a rate of 15 frames per second looped back and forth continuously during the perceptual task. The amplitude of the rotational movement was 39 deg. A longer sequence was not used because in that case the surrounding cells would have overlapped the leukaemia cell. The size of the reconstruction was $539 \times 412$ pixels corresponding to $14.5 \times 11.1 \mathrm{~cm}^{2}$ and 9.2 x $7.1 \mathrm{deg}^{2}$ at a viewing distance of $90 \mathrm{~cm}$.

\section{RESULTS}

Using the stereoscopic depth-matching tool the observers produced matches for the holographic coil and cell objects. These results are also shown in Fig. 1. When the coil was presented as non-stereo and static, the matched stereoscopic depth was only $1.73 \mathrm{~cm}$ while the true depth of the depicted object was $9.9 \mathrm{~cm}$. Thus, the matched stereoscopic depth was hugely underestimated. Adding motion to the non-stereo presentation of the same object increased the matched stereoscopic depth to $3.7 \mathrm{~cm}$, which was still grossly underestimated. The difference between the depth estimates with and without motion was statistically significant (MannWhitney test: $U=396.5 ; p=0.0204)$. Stereoscopic presentation of the coil object as a static image or with back and forth rotational motion produced somewhat overestimated matched stereoscopic depths on average. These were $12.4 \mathrm{~cm}$ for the static stereoscopic presentation and $11.3 \mathrm{~cm}$ for stereoscopic presentation with motion. The latter was closer to the real depth value of $9.9 \mathrm{~cm}$. The difference between these results was quite small but statistically significant ( $U=172.5$; $\mathrm{p}=0.0034$ ).

The cell object appeared to be relatively blurred in comparison to the coil object. This could be seen in the amplitude spectra of the objects. The spectrum of the cell object declined much more rapidly than the spectrum of the coil object. Thus, we hypothesise that the lack of high spatial frequencies could have made the computation of stereo

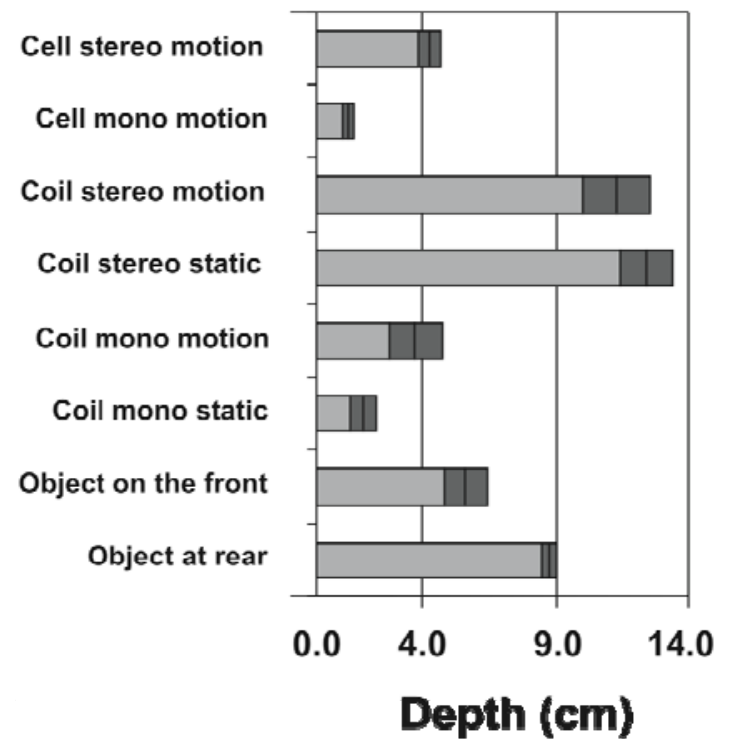

Fig. 1. Matched stereoscopic depths (cm) for different objects in different conditions. The two dark grey areas at the right ends of the bars show the \pm 1 standard error.

correspondence more difficult and, therefore, reduced stereoscopic depth information. The reduced stereoscopic information could have lead to the inability to see depth in the static stereoscopic cell object. This might have been compensated for by motion parallax, which could explain the additive effect of motion on perceived depth in the presence of stereo. In order to test this hypothesis we performed an additional experiment where we measured perceived stereoscopic depth for the coil object blurred by various amounts. The blurring was produced by Fourier filtering with circularly symmetric Butterworth low-pass filters of various cut-off frequencies [5].

The results for the low-pass filtered static stereo coil object and stereo coil object with motion showed that considerable blurring had a clear effect on the matched stereoscopic depth for the static stereoscopic image. The effect became gradually less prominent as the cut-off frequency of the filter increased. However, when the stereoscopic presentation was combined with motion the effect of blur was considerably smaller. This result supports the hypothesis that the absence of high spatial frequencies may explain the additivity of stereo and motion in perceived depth for the cell object. 


\section{DISCUSSION}

The results showed that in non-stereo presentation of hologram reconstructions motion could increase perceived depth in comparison to static image presentation. Further, stereoscopic presentation increased perceived depth considerably in comparison to non-stereoscopic presentation if stereoscopic cues are strong. Considerable image blur weakened depth perception so that perceived depth became small. Thus, very low spatial frequencies, at least relative to object size, do not seem to mediate stereo information efficiently. The results obtained by using band-pass filtered images showed that both at very low and very high spatial frequencies perceived depth was reduced. Motion seemed to alleviate this effect, however.

The present study clearly demonstrates that the viewing method can have a great effect on depth perception of numerical hologram reconstructions presented on conventional displays. If depth cues are weak in holographic reconstructions, it will probably be highly beneficial for the observer to have a possibility to use both motion and stereo presentation in order to maximise $3 \mathrm{D}$ information. This conclusion is particularly relevant in such cases where the objects viewed are novel to the observer - as was in the case of the microscopic cell object of this study - and, therefore, the interpretation of image features and monocular depth cues can be difficult.

\section{ACKNOWLEDGEMENTS}

The authors would like to thank Dr Emmanouil Darakis for capturing the hologram sequence of the coil object. This research received funding from the European Community's Seventh Framework Programme FP7/20072013 under grant agreement no. 216105 (Real3D).

\section{REFERENCES}

[1] T. Lehtimäki and T.J. Naughton, "Stereoscopic viewing of digital holograms of real-world objects," presented at Capture, Transmission and Display of 3D Video, article no. 39, Kos, Greece, 7 - 9 May 2007.

[2] C. Reichle, T. Müller, T. Schnelle, G. Fuhr, "Electro-rotation in octopole micro cages," J. Physics D: Appl. Physics 32, 2128 - 2135 (1999).

[3] P. Marquet, B. Rappaz, P.J. Magistretti, E. Cuche, Y. Emery, T. Colomb, C. Depeursinge, "Digital holographic microscopy: a noninvasive contrast imaging technique allowing quantitative visualization of living cells with subwavelength axial accuracy," Optics Letters 30, 468-470 (2005).

[4] T. Colomb, F. Montfort, J. Kühn, N. Aspert, E. Cuche, A. Marian, F. Charrière, S. Bourquin, P. Marquet, C. Depeursinge, "Numerical parametric lens for shifting, magnification and complete aberration compensation in digital holographic microscopy," J. Opt. Soc. Am. A 23, 3177-3190 (2006).

[5] R.C. Gonzalez and E.E. Woods, Digital Image Processing. 2nd Edition. (Prentice-Hall, Inc. 2002). 